\title{
Optimization of working cathode position in sleeve-type bioelectrochemical system with inner chamber/outer chamber for azo dye treatment
}

\author{
Fanying Kong a , Aijie Wang ${ }^{\mathrm{a}, \mathrm{b}, *}$, Hong-Yu Ren ${ }^{\mathrm{a}}$ \\ ${ }^{a}$ State Key Laboratory of Urban Water Resource and Environment, School of Municipal and Environmental Engineering, Harbin Institute of Technology, Harbin 150090, China \\ ${ }^{\mathrm{b}}$ Research Center for Eco-Environmental Sciences, Chinese Academy of Sciences, Beijing 100085, China
}

\section{H I G H L I G H T S}

-Working position in sleeve-type BES was evaluated and optimized for decolorization.

- Cathode in outer chamber performed better both at $V_{\text {cathode }}: V_{\text {anode }}=1: 1$ and 3:1.

- Current and EIS analysis showed outer cathode benefited proton/electron transfer.

- Lower decolorization with increased $V_{\text {cathode }}$ might be due to less substrate supply.

- It would be further improved through increasing substrate concentration at anode.

\section{A R T I C L E I N F O}

Article history:

Received 2 August 2015

Received in revised form 8 September 2015

Accepted 9 September 2015

\section{Keywords:}

Bioelectrochemical system (BES)

Sleeve-type configuration

Working position

Decolorization

Azo dye

\begin{abstract}
A B S T R A C T
In this study, the optimization of working cathode position in sleeve-type bioelectrochemical system (BES) was evaluated with inner/outer chamber for azo dye decolorization. Results showed that the working position in outer chamber performed better with decolorization efficiencies of $97.8 \pm 2.1 \%(7 \mathrm{~h})$ and $94.0 \pm 2.3 \%(16 \mathrm{~h})$ than that in inner chamber as the volume ratio $V_{\text {cathode }}: V_{\text {anode }}=1: 1$ and $3: 1$, respectively. The current and electrochemical impedance spectroscopy (EIS) analysis indicated that the proton/electron transfer and anolyte diffusion could be improved using outer chamber as working position. The decolorization with increased volume ratio could be further improved through the strategy of increasing substrate concentration, which would provide enough electrons and decrease diffusion resistance, further improving the whole performance with increased outer cathode volume. It has the great potential in sleeve-type configuration application and would create more challenges for process optimization and maintenance.
\end{abstract}

(c) 2015 Elsevier Ltd. All rights reserved.

\section{Introduction}

Bioelectrochemical system (BES) as a novel and promising approach, is very suitable for treating wastewater rich in unsaturated bonds (such as azo dye, antibiotics, pentachlorophenol, nitrobenzene, and heavy metals) at cathode, being established world widely as an alternative wastewater treatment process (Huang et al., 2012; Kong et al., 2015a, 2013; Mu et al., 2009a, 2009b; Tao et al., 2014). It can catalyze the degradation of organic matter at anode to provide electrons and protons for the cathode reduction and to reduce overpotential (Wang and Ren, 2013).

\footnotetext{
* Corresponding author at: State Key Laboratory of Urban Water Resource and Environment, School of Municipal and Environmental Engineering, Harbin Institute of Technology, Harbin 150090, China. Tel./fax: +86 45186282195.

E-mail address: waj0578@hit.edu.cn (A. Wang).
}

Considering the separation between the oxidation and the reduction reactions, dual chamber structures are required as suitable BES configuration to increase the antitoxic feasibility when the reactions take place in the processing of contaminants from wastewaters, resulting in a possibly whole range of application (Huang et al., 2012; Kong et al., 2015a, 2013; Mu et al., 2009a, 2009b; Tao et al., 2014).

It has reported that the effectiveness of BES will be highly dependent on the design choice (Li et al., 2014; Wang and Ren, 2013; Wang et al., 2012). The favorable BES application in the future will be obtained in the BES reactor with the lowest internal resistance, thus considerable effort should be applied to reduce the internal resistance (Sleutels et al., 2012). Recent research has modified the configuration of BES to be sleeve-type that with large area and small distance between anode and cathode, reducing the electrons transfer resistance and mass transfer resistance 
(Kong et al., 2013). Its advantages have been demonstrated in some cases, for example desalination, decolorization and the treatment of chromium (Huang et al., 2010; Jacobson et al., 2011; Kong et al., 2015b, 2015c). These studies indeed provided the feasibility of the BES with sleeve-type configuration to treat wastewater, however, as a sustainable and environment-friendly technology, the practical application of BES is strongly limited by their configuration optimum and the assembled optimization for operation (Ge et al., 2014; Gil-Carrera et al., 2011; Ping and He, 2013; Raman and Lan, 2012; Uría et al., 2012), which could provide important theory for further scale-up. The emerging alternative application of BES for recalcitrant pollutants has not yet been evaluated in optimizing the state of working position, with inner chamber or outer chamber. The working position in inner chamber or outer chamber can help us, not only to gain better understanding of the BES configuration, but also enable us to influence reaction properties.

Thus, aiming to find the best arrangement in the sleeve-type BES reactor for azo dye treatment, this work evaluated and optimized the working cathode position of outer or inner chamber at $V_{\text {cathode }}: V_{\text {anode }}$ of $1: 1$. Further optimization was also investigated in the increased volume ratio of $3: 1$. This will provide a theoretical basis and reference for future practical application.

\section{Methods}

\subsection{Configuration of sleeve-type BES}

The BES reactor was constructed in sleeve-type configuration as previously described with an inner tube and an outer tube using Perspex (Kong et al., 2013). The inner tube with full of holes was identified as the inner chamber when cation exchange membrane (Ultrex CMI7000, Membranes International Inc., USA) was pasted. The space between the inner tube and outer tube was identified as the outer chamber. As shown in Fig. 1, the working position of BES-1\# and BES-3\# was the outer chamber, with cathode electrode placed into the outer chamber and anode in the inner chamber. While on the contrary, the working position of BES-2\# and BES$4 \#$ was the inner chamber, with cathode in the inner chamber and anode in the outer chamber. The cathode chamber and anode chamber of BES-1\# and BES-2\# have the same volume with cathode and anode volume ratio of $1: 1\left(V_{\text {cathode }}: V_{\text {anode }}=1: 1\right)$, and the

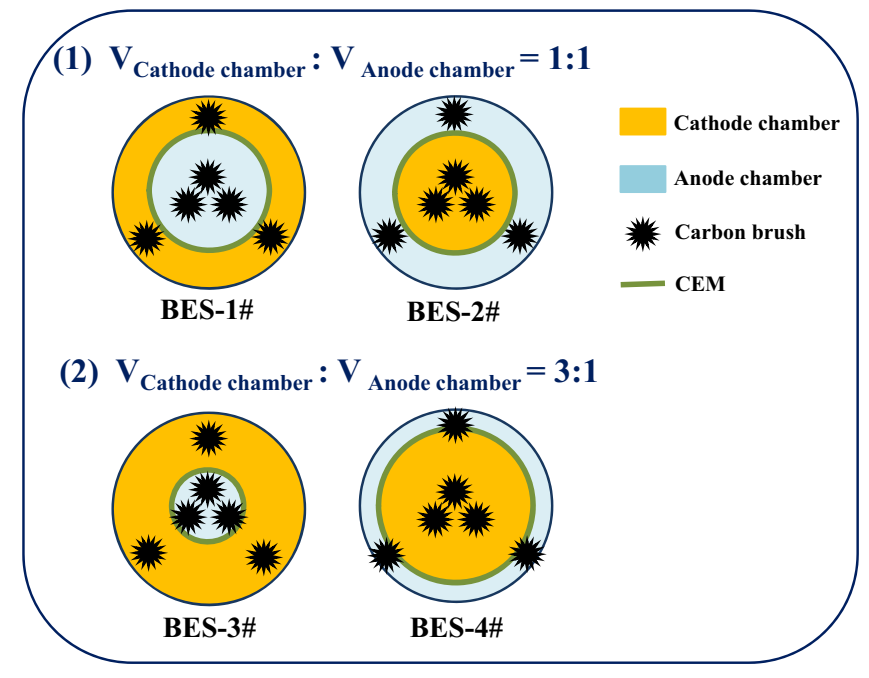

Fig. 1. Configuration and operation of sleeve-type bioelectrochemical reactor at $V_{\text {cathode }}: V_{\text {anode }}=1: 1$ and $3: 1$. In BES-1\# and BES-3\#, the working position was the outer chamber, with cathode placed into the outer chamber and anode in the inner chamber. In BES-2\# and BES-4\#, the working position was the inner chamber, with cathode in the inner chamber and anode in the outer chamber.
BES-3\# and BES-4\# with $V_{\text {cathode }}: V_{\text {anode }}=3: 1$ were constructed to evaluate the influence of increased cathode volume on azo dye treatment. Both anode and cathode electrode materials were carbon brush (ID $2 \mathrm{~cm} \times \mathrm{L} 8 \mathrm{~cm}$ ) displaced to be surrounding deployment as described previously (Fig. 1) (Kong et al., 2014). The experiments were performed with the applied voltage of $0.5 \mathrm{~V}$ supplied with a DC power supply. For the reason that the anode potential was sustained at $-0.4 \mathrm{~V}$ or even lower with acetate as the substrate, only with the applied voltage of $0.5 \mathrm{~V}$, the cathode potential could be kept at $-0.9 \mathrm{~V}$, which was feasible for AO7 reduction decolorization (Mu et al., 2009a; Zille et al., 2004).

\subsection{Operation of experiments}

Sodium acetate (NaAc) was the substrate in the anode chamber used as the sole electron donor, and azo dye (acid orange 7, AO7) was treated in the cathode chamber used as the sole electron accepter. In principle, the acetate could produce protons and electrons at anode that transferred to the cathode for the azo bond cleavage of azo dye. Anode and cathode were inoculated with anaerobic sludge obtained from wastewater treatment plant in Harbin, China. The anolyte was NaAc $1 \mathrm{~g} \mathrm{~L}^{-1}$ (expect for the effect of substrate concentration), $\mathrm{KCl} 0.13 \mathrm{~g} \mathrm{~L}^{-1}, \mathrm{NH}_{4} \mathrm{Cl} 0.31 \mathrm{~g} \mathrm{~L}^{-1}$, PBS $50 \mathrm{mM}$, trace element $1 \mathrm{~mL} \mathrm{~L}^{-1}$ and Wolf's vitamin $1 \mathrm{~mL} \mathrm{~L}^{-1}$ (with conductivity of $7.0 \pm 0.5 \mathrm{mS} \mathrm{cm}^{-1}$ ). The catholyte was $\mathrm{AO} 7$ $100 \mathrm{mg} \mathrm{L}^{-1}, \mathrm{KCl} 0.13 \mathrm{~g} \mathrm{~L}^{-1}, \mathrm{NH}_{4} \mathrm{Cl} 0.31 \mathrm{~g} \mathrm{~L}^{-1}$, PBS $50 \mathrm{mM}$, trace element $1 \mathrm{~mL} \mathrm{~L}^{-1}$ and Wolf's vitamin $1 \mathrm{mLL}^{-1}$ (with conductivity of $7.0 \pm 0.5 \mathrm{mS} \mathrm{cm}^{-1}$ ). The anolyte and catholyte were refilled with fresh medium each $24 \mathrm{~h}$. All tests were conducted and repeated at least five times. Sleeve-type bioelectrochemical reactors with inner chamber or outer chamber as working chamber were established for decolorization of azo dye. BES-1\# and BES-2\# with $V_{\text {cath- }}$ ode $: V_{\text {anode }}=1: 1$ were investigated to evaluate the effect of working cathode position on the BES decolorization performance with the same chamber volume of cathode and anode. Meanwhile, BES-3\# and BES-4\# with $V_{\text {cathode }}: V_{\text {anode }}=3: 1$ were also compared to study the effect of working position on sleeve-type BES performance if increased the volume ratio of working chamber. In order to identify if the electrons supply or anolyte diffusion that affected the performance with outer cathode in BES-3\# at $V_{\text {cathode }}: V_{\text {anode }}=3: 1$ and to improve the performance for scale-up, experiments were performed with increased acetate concentration from 1000 to $3000 \mathrm{mg} \mathrm{L}^{-1}$ in the inner anode chamber.

\subsection{Analytical methods}

The AO7 concentration was measured at $\lambda_{\max }=484 \mathrm{~nm}$ by UVvis scanning spectrophotometer (Shimadzu UV2550, Japan). Anode and cathode as well as the reference electrode (saturated calomel reference electrode, SCE, model-217, Shanghai Precise. Sci. Instru. Co., Ltd., China; 0.247 V vs. standard hydrogen electrode, SHE) were connected to a data acquisition unit (keithley 2700, Keithley Co., Ltd., USA) with external resistance of $20 \Omega$ to record electrode potentials and current. Electrochemical impedance spectroscopy (EIS) was carried in a frequency range of $10^{5}-0.01 \mathrm{~Hz}$ with a perturbation signal of $10 \mathrm{mV}$ using electrochemical workstation (model-660D, CH Instruments Inc., USA).

\section{Results and discussion}

3.1. The optimization of working cathode position in sleeve-type BES with $V_{\text {cathode }}: V_{\text {anode }}=1: 1$

In order to investigate the decolorization performance with different working positions in the sleeve-type BES, two BES reactors 
were firstly performed and compared at the volume ratio $V_{\text {cathode }}: V_{\text {anode }}$ of $1: 1$ to ensure the comparability, including BES-1\# with outer chamber as the working position (i.e. cathode electrodes were placed in the outer chamber) and BES-2\# with inner chamber as the working position (i.e. cathode electrodes were placed in the inner chamber) (Fig. 1). They were analyzed in terms of decolorization, current and EIS to obtain the decolorization behavior of sleeve-type BES with different working positions and the reason leading to the different results.

\subsubsection{Decolorization performance}

For BES with $V_{\text {cathode }}: V_{\text {anode }}=1: 1$, results showed that the working cathode position in the outer chamber took obvious advantages compared to that in the inner chamber. The decolorization efficiency in the BES-1\# with outer cathode was $97.8 \pm 2.1 \%$ at $7 \mathrm{~h}$, which was higher than that in BES-2\# with inner cathode $(90.9 \pm 3.4 \%)$, and it would take $12 \mathrm{~h}$ to reach the similar decolorization efficiency of $97.2 \pm 2.8 \%$ (Fig. 2A). HPLC analysis indicated that sulfanilic acid (SA) and 1-amino-2-naphthol (AN) were the dominant products of the AO7 decolorization at the cathode, demonstrating the cleavage of the azo bond of azo dye in BES. The formation efficiencies of SA were higher than $90 \%$ as over 98\% AO7 decolorized simultaneously (data not shown). In order to investigate the dynamic characteristics for AO7 decolorization in BES, the decolorization behavior with different working positions was also analyzed in terms of kinetic study by fitting AO7 concentrations as a function of time. Results exhibited that $\mathrm{AO} 7$ decolorization in the BES conformed to the first-order model, and the corresponding reaction rate constant $k\left(\mathrm{~h}^{-1}\right)$ was $0.644 \mathrm{~h}^{-1}$ in the BES-1\#, which was 1.6-fold higher than BES-2\# with inner cathode $\left(0.404 \mathrm{~h}^{-1}\right)$ (Fig. 2A), further indicating the better decolorization in the BES-1\# with outer cathode. Thus, the outer working position was based on a kinetic mechanism that accelerated the reaction rate, which was a significant kinetic factor in the BES (Liang et al., 2013).

These different decolorization results between BES-1\# and BES2\# showed that the working position in the sleeve-type BES played an important role in the wastewater treatment performance, and working position in the outer chamber was better than that in inner chamber at $V_{\text {cathode }}: V_{\text {anode }}=1: 1$.

\subsubsection{Current}

Current is a key parameter to describe conversion rate (Zhao et al., 2009). It was observed that the BES-1\# generated a peak current of $0.022 \mathrm{~A}$, while the peak current of BES-2\# was $0.012 \mathrm{~A}$, which was 1.8 -fold decrease (Fig. 2B). According to that current was related to the electron flows, which were inherent to the microbial metabolism that microorganisms transfer electrons from an electron donor to an electron acceptor (Zhao et al., 2009), it was expected that a higher current might be attributed to the efficient electrolyte transport and enhanced electron transfer between the microbial biofilm and the electrode. These current results strongly suggested that the BES-1\# with outer working position enabled superior performance due to an increase in electron transfer with current generation, which would result in faster azo dye decolorization (Fig. 2A).

Moreover, the current of BES-1\# reached the maximum at the beginning and then gradually decreased (Fig. 2B). It has reported that the peak current was observed due to the favorable oxidation of the electron donor (Zhao et al., 2009), thus, the faster substrate degradation at inner anode that provided enough electrons and protons for the $\mathrm{AO7}$ reduction at cathode, resulting in the maximum current in a short time. Moreover, the maximum current cannot be sustained because of the rapidly depleted donor near the electrode (Zhao et al., 2009), leading to the gradual decrease as shown in Fig. 2B. While for the BES-2\#, the substrate degradation of anode was in the outer chamber, which might be constricted by the anolyte diffusion, performing the gradual increase in current. Similar to BES-1\#, the subsequent decrease might be resulting from the finish of substrate degradation and diminished AO7.

These results indicated that the working position in the sleevetype BES with $V_{\text {cathode }}: V_{\text {anode }}=1: 1$ could affect the current with wastewater in the outer chamber or inner chamber. The working position in the outer chamber performed better performance in electron transfer, probably due to the faster substrate degradation at inner anode and enough electrons and protons transferred to the cathode for decolorization.

\subsubsection{EIS}

EIS analysis is another principal method to examine the electrochemical behavior of BES configuration (He and Mansfeld, 2009). In order to further analyze the reason of improved BES performance, EIS of BES-1\# and BES-2\# had been compared and discussed in the total resistances (Fig. 2C) and their individual components (Fig. 2D). Fig. 2C showed that EIS results of BES-1\# and BES-2\# were different as operated with different working positions. The total resistance of BES-1\# was $35.8 \Omega, 1.9$-fold lower than that of BES-2\# (66.9 $\Omega$ ). The advantage of BES-1\# with this lower total resistance was due to the combination of lower anode resistance and cathode resistance. Results showed that the anode resistance of BES-1\# was $28.2 \Omega, 31.1 \%$ lower than that of BES-2\# (40.9 $\Omega$ ), while the cathode resistance of BES-1\# was $7.6 \Omega, 70.8 \%$ lower than that of BES-2\# (26.0 $\Omega$ ) (Fig. 2 C). It can be seen that the sleeve-type BES with working position in the outer chamber could reduce anode resistance and cathode resistance of BES reactor, especially cathode resistance.

According to that resistance was the ability of circuit element to hinder the flow of electrons (Kashyap et al., 2014), the anode resistance and cathode resistance of BES-1\# and BES-2\# were analyzed in detail through the components of resistance, including ohmic resistance $\left(R_{\mathrm{ohm}}\right)$, charge transfer resistance $\left(R_{\mathrm{ct}}\right)$ and diffusion resistance $\left(R_{\text {diff }}\right)$ to find the reason of difference performance with different working positions (Fig. 2D). Firstly, for the anode resistances in BES-1\# and BES-2\#, the lower anode resistance of BES$1 \#$ might be due to the lower $R_{\mathrm{ct}}$ and $R_{\mathrm{diff}}$ of anode compared with BES-2\#. The $R_{\mathrm{ct}}$ of anode in BES-1\# and BES-2\# was 9.9 and $15.5 \Omega$, respectively, while the corresponding $R_{\text {diff }}$ was 13.2 and $19.4 \Omega$ (Fig. 2D). The lower charge transfer resistance and diffusion resistance of anode in BES-1\# demonstrated the enhanced charge transfer at anode and anolyte diffusion in the anode chamber. The improved anolyte diffusion would accelerate the substrate degradation and promote the produce of electrons that provided to cathode, resulting in the improved decolorization (Fig. 2A). Secondly, for the cathode resistances in BES-1\# and BES-2\#, it indicated that the different cathode resistances in BES-1\# and BES-2\# were caused by the interaction of $R_{\mathrm{ohm}}, R_{\mathrm{ct}}$, and $R_{\mathrm{diff}}$. The $R_{\mathrm{ohm}}, R_{\mathrm{ct}}$, and $R_{\text {diff }}$ of cathode in BES-1\# were $0.8,2.9$ and $3.9 \Omega$, respectively, while those in BES-2\# were 7.5, 6.8 and $11.7 \Omega$ (Fig. 2D). Thus, the better BES-1\# with working position in the outer chamber might be associated with the better proton/electron transfer related to the substrate degradation at anode. With the inner anode in BES$1 \#$, the substrate was concentratedly degraded in the center, with protons transferred from the center to the surrounding CEM, which making the relative large surface area of proton transfer. Moreover, the concentrated substrate in the center might have decreased diffusion resistance, which was benefit to the substrate degradation at anode and would promote electron supply for the cathode, finally resulting in the good decolorization performance (Fig. 2A).

These results indicated that in the sleeve-type BES with $V_{\text {cathode }}: V_{\text {anode }}$ of $1: 1$, the working position in the outer chamber was better than the inner chamber, which could improve the charge transfer and substrate diffusion in the anode chamber, and probably 

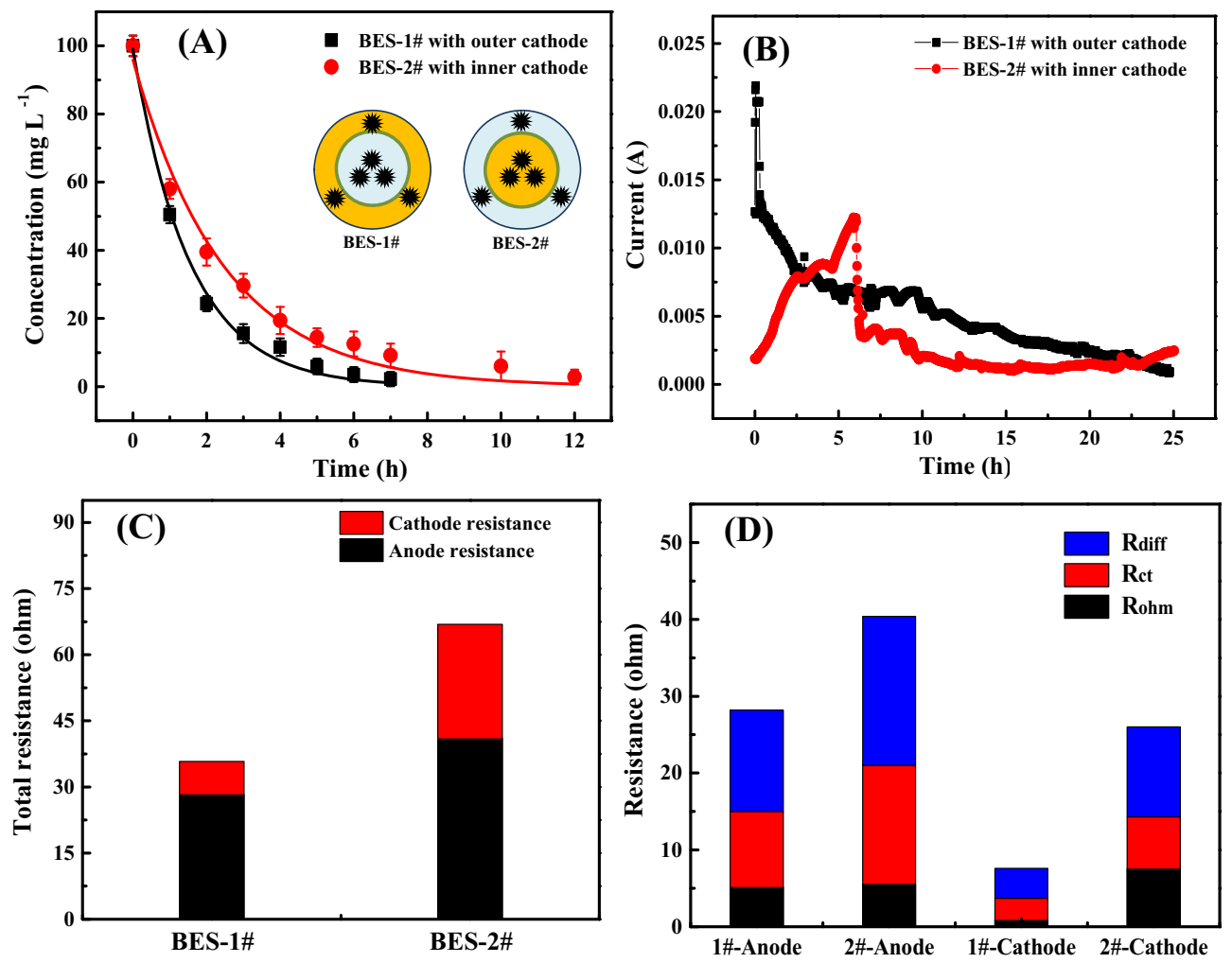

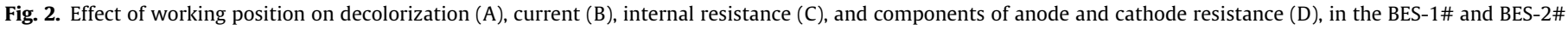
with cathode and anode volume ratio $V_{\text {cathode }}: V_{\text {anode }}=1: 1$.

enhance the supply of electrons and protons for AO7 reduction at cathode.

\subsection{The optimization of working cathode position in sleeve-type BES with $V_{\text {cathode }}: V_{\text {anode }}=3: 1$}

The optimization of working positions (BES-3\# with outer cathode chamber and BES-4\# with inner cathode chamber) was also evaluated at increasing $V_{\text {cathode }}: V_{\text {anode }}$ of 3:1 to study the effect of working positions in BES with increased working volume.

\subsubsection{Decolorization performance}

In the BES with increasing volume ratio of $V_{\text {cathode }}: V_{\text {anode }}=3: 1$, BES-3\# with outer cathode exhibited the better decolorization $(94.0 \pm 2.3 \%$ at $16 \mathrm{~h})$, followed by BES-4\# with inner cathode $(84.2 \pm 4.1 \%$ at $16 \mathrm{~h})$ (Fig. $3 \mathrm{~A})$. The reaction rate constant $k$ for BES-3\# was $0.227 \mathrm{~h}^{-1}$, which was 1.9 -fold higher than BES-4\# with inner cathode $\left(0.117 \mathrm{~h}^{-1}\right)$ (Fig. $\left.3 \mathrm{~A}\right)$. It can be seen that the BES with outer working position took obvious advantage of decolorization in comparison with BES with inner working position at $V_{\text {cathode: }}$ $V_{\text {anode }}=3: 1$. Based on that the $k$ of BES-1\# was 1.6 -fold higher than that of BES-2\# (Fig. 2A) and the $k$ of BES-3\# was 1.9-fold higher than that of BES-4\# (Fig. 3A), this increase of 1.6-fold to 1.9-fold indicated that the BES with outer working position could play more predominant priority as $V_{\text {cathode }}: V_{\text {anode }}$ increased within a certain range.

In all the BES reactors, BES-1\#, BES-2\#, BES-3\# and BES-4\# removed $97.8 \pm 2.1 \%(7 \mathrm{~h}), 97.2 \pm 2.8 \%(12 \mathrm{~h}), 94.0 \pm 2.3 \%(16 \mathrm{~h})$, and $91.2 \pm 3.6 \%$ ( $24 \mathrm{~h}$ ), respectively. The time needed for $90 \%$ decolorization was $4,6,12$, and $24 \mathrm{~h}$, respectively (Figs. 2A, 3A, and Table 1). It can be seen that the BES-1\# with outer working position $\left(V_{\text {cathode }}: V_{\text {anode }}=1: 1\right.$ ) exhibited the best decolorization performance, followed by BES-2\# with inner working position $\left(V_{\text {cathode }}: V_{\text {anode }}=1: 1\right)$, BES-3\# with outer working position ( $V_{\text {cathode }}: V_{\text {anode }}=3: 1$ ) and then BES-4\# with inner working position ( $V_{\text {cathode }}: V_{\text {anode }}=3: 1$ ). These results indicated that the decolorization was dependent on the working position in the sleeve-type BES with inner chamber and outer chamber. It should be noted that along with the increased volume ratio, the decolorization was decreased. Take BES with outer working position for example, the reaction rate kinetics were $0.644 \mathrm{~h}^{-1}$ for BES-1\# with $V_{\text {cathode }}: V_{\text {anode }}=1: 1$ and $0.227 \mathrm{~h}^{-1}$ for BES-3\# with $V_{\text {cathode }}$ : $V_{\text {anode }}=3: 1$ (Table 1 ). Considering that substrate degradation at anode would supply protons and electrons for decolorization at cathode, the less effective decolorization might be related to the substrate in a short supply at anode for more AO7 decolorization when the cathode volume increased.

\subsubsection{Current}

BES-3\# generated a higher peak current of 0.016 A than BES-4\# (0.008 A) (Fig. 3B). The results also demonstrated that the BES with outer working position exhibited better performance than that with inner one. The current of BES-3\# and BES-4\# was increased gradually to the maximum current and then decreased. The lower current of BES in the early period (before $3.5 \mathrm{~h}$ for BES-3\# and $5.6 \mathrm{~h}$ for BES-4\#) might be limited by the substrate degradation. If the substrate degradation feeding electrons for the cathode was sufficient at the beginning of the process, it would be expected to be a sharp current increase as the substrate addition (Wang et al., 2011). The substrate degradation at anode might be depending upon the concentration of substrate and the diffusion of substrate (Montpart et al., 2015; Velasquez-Orta et al., 2011; Wang et al., 2013). These results also showed that the improved decolorization and enhanced current were not only caused by the anodic reaction, 

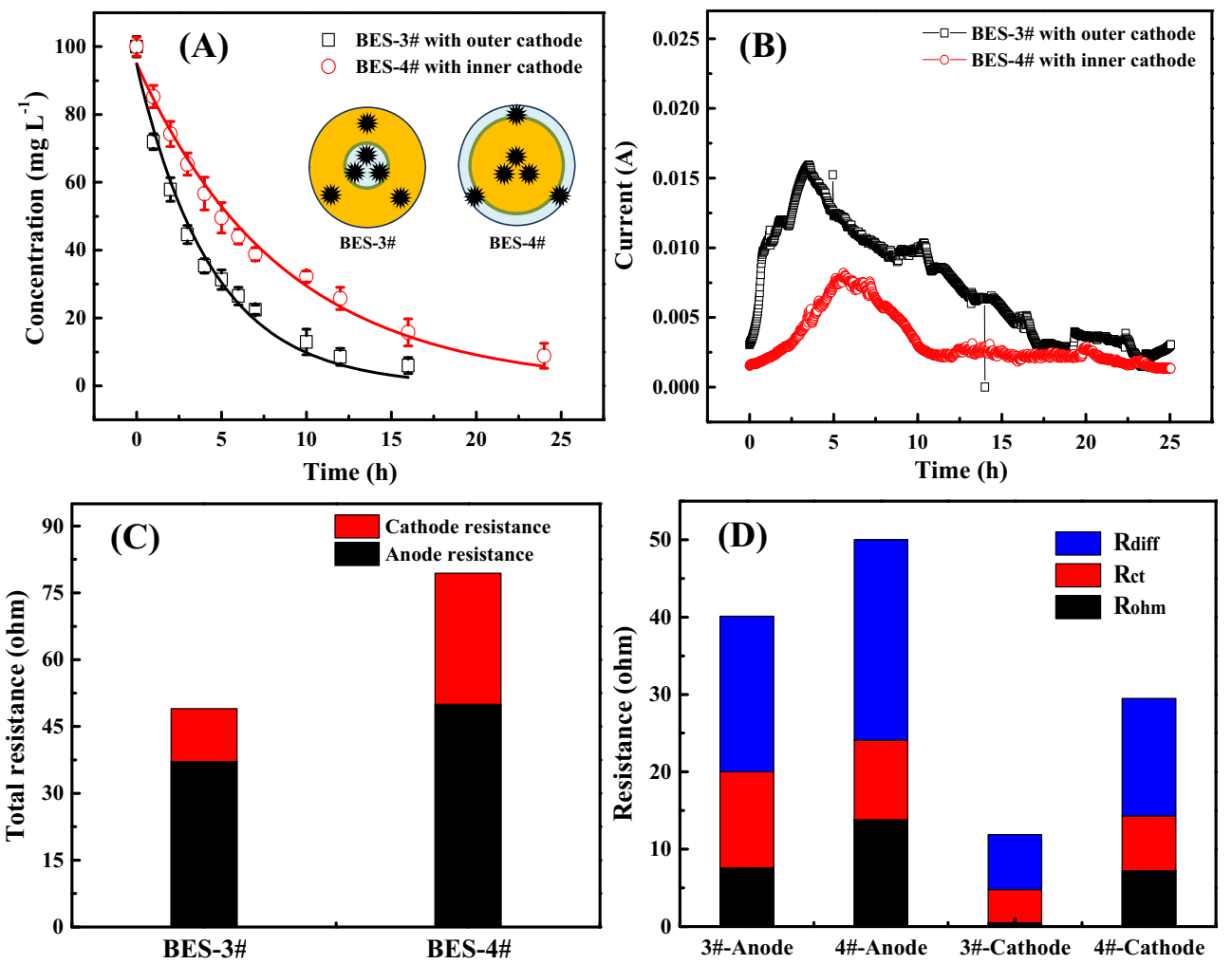

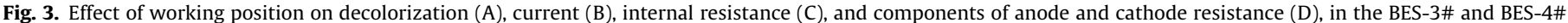
with cathode and anode volume ratio $V_{\text {cathode }}: V_{\text {anode }}=3: 1$.

Table 1

Performance comparison of BES-1\#, BES-2\#, BES-3\# and BES-4\#.

\begin{tabular}{|c|c|c|c|c|}
\hline \multirow[t]{2}{*}{ Performance } & \multicolumn{2}{|c|}{$V_{\text {cathode }}: V_{\text {anode }}=1: 1$} & \multicolumn{2}{|c|}{$V_{\text {cathode }}: V_{\text {anode }}=3: 1$} \\
\hline & BES-1\# & BES-2\# & BES-3\# & BES-4\# \\
\hline Decolorization efficiency (\%) & $97.8 \pm 2.1(7 \mathrm{~h})$ & $97.2 \pm 2.8(12 \mathrm{~h})$ & $94.0 \pm 2.3(16 \mathrm{~h})$ & $91.2 \pm 3.6(24 \mathrm{~h})$ \\
\hline$k\left(\mathrm{~h}^{-1}\right)$ & 0.644 & 0.404 & 0.227 & 0.121 \\
\hline Maximum current (A) & 0.022 & 0.012 & 0.016 & 0.008 \\
\hline Total resistance $(\Omega)$ & 35.8 & 66.9 & 49.0 & 79.4 \\
\hline Anode resistance $(\Omega)$ & 28.2 & 40.9 & 37.1 & 50.0 \\
\hline Cathode resistance $(\Omega)$ & 7.6 & 26.0 & 11.9 & 29.4 \\
\hline
\end{tabular}

but by the reaction rate of the azo dye at cathode surface which decisively affected on the current.

\subsubsection{EIS}

The total resistance of the BES-3\# with the outer working position $(49.0 \Omega$ ) was also lower than the BES-4\# with inner one (79.4 $\Omega$ ) as the $V_{\text {cathode }}: V_{\text {anode }}$ increased to $3: 1$ (Fig. 3C). It was similar to the comparison of BES-1\# and BES-2\# in the volume ratio of $1: 1$, further indicating the improved BES performance with outer cathode in the sleeve-type configuration. This also pointed out that as the volume ratio of cathode and anode chamber increased in a certain range, the working electrode placed in the outer chamber was more favorable. Moreover, the anode resistances of BES-3\# and BES-4\# were 37.1 and $50.0 \Omega$, respectively, and the corresponding cathode resistances were 11.9 and $29.4 \Omega$ (Fig. 3C). It indicated that both the anode resistance and cathode resistance in BES-3\# were lower than those in BES-4\#. Thus, the performance of BES-3\# was improved by the combined enhancement of anode and cathode. It would be further testified in detail by the analysis of components of anode resistance and cathode resistance as showed in Fig. 3D.
Results indicated that the different resistances between the two reactors were mainly resulted from the different $R_{\mathrm{ohm}}$ and $R_{\mathrm{diff}}$. The $R_{\text {ohm }}$ of anode in BES-3\# and BES-4\# was 7.6 and $13.8 \Omega$, respectively, and the corresponding $R_{\text {diff }}$ was 20.1 and $25.9 \Omega$. Meanwhile, the $R_{\text {ohm }}$ of cathode in BES-3\# and BES-4\# was 0.5 and $7.2 \Omega$, respectively, and the corresponding $R_{\text {diff }}$ was 7.1 and $15.2 \Omega$ (Fig. 3D). The data suggested that the BES with outer working position caused a remarkable decrease in the $R_{\mathrm{ohm}}$ and $R_{\mathrm{diff}}$, indicating a better performance could be obtained through the optimization of working position and the BES with outer cathode was a favorable way for sleeve-type BES application in wastewater treatment. According to that the $R_{\mathrm{ohm}}$ was related to the electron transfer resistance through the electrode material and proton transfer in solution or through the membrane (He and Mansfeld, 2009), the BES-3\# and BES-4\# might be with different electrode distance and proton transfer distance due to the different diameters of inner tube and outer tube at the same $V_{\text {cathode }}: V_{\text {anode }}=3: 1$ (Fig. 1 ), which resulting in the different $R_{\text {ohm }}$. The different diameters also led to the different electrolyte diffusion, which resulting in the different diffusion resistance (Fig. 3D).

In conclusion, compared with BES with inner working position, the substrate diffusion in the anode chamber of BES with outer 
working position could be improved, accelerating the transport of substrate between solution and anode and then improving the substrate degradation which supplied protons and electrons for decolorization at cathode. Thus, the BES performance with outer working position performance might be further optimized by increasing the substrate concentration at anode or the electrolyte diffusion.

\subsection{Further improvement through increasing substrate concentration}

The above results demonstrated that the best decolorization performance within $7 \mathrm{~h}$ was observed in the BES with outer working position $(97.8 \pm 2.1 \%)$ in comparison with inner one $(90.9 \pm 3.4 \%)$ at $V_{\text {cathode }}: V_{\text {anode }}=1: 1$ (Fig. $\left.2 \mathrm{~A}\right)$. As increasing the volume ratio to be $V_{\text {cathode }}: V_{\text {anode }}=3: 1$, the BES with outer working position $(77.6 \pm 1.3 \%)$ was also taken superiority to the BES with inner one $(61.2 \pm 1.9 \%)$ (Fig. $3 \mathrm{~A})$. As can be seen, BES performance was inhibited as increasing the working volume ratio, which needed to be further improved to construct the application of BES with sleeve-type configuration. The current and EIS analysis indicated that the electron transfer and diffusion of anode were the limiting factors that affected the performance of BES with the relative large cathode volume (Figs. 2 and 3). Meanwhile the reason that resulting in the decreased decolorization might be due to the limited supply of electrons that provided by substrate degradation at anode. Thus, much effort should be made in improving its performance through increasing the substrate concentration.

The decolorization as a function of time was well described by first-order kinetics with different substrate concentrations from 1000 to $3000 \mathrm{mg} \mathrm{L}^{-1}$ (Fig. 4A). Although the decolorization efficiencies with different substrate concentrations were almost the same at $24 \mathrm{~h}$ (about 98\%), the corresponding reaction rate constants $k\left(\mathrm{~h}^{-1}\right)$ was $0.227,0.399$ and $0.319 \mathrm{~h}^{-1}$ for 1000,2000 , and
$3000 \mathrm{mg} \mathrm{L}^{-1}$, respectively (Fig. 4A). These results indicated that initial substrate concentrations in the anode chamber had a significant impact on the AO7 decolorization in the cathode chamber, and $2000 \mathrm{mg} \mathrm{L}^{-1}$ was the best substrate concentration as the $V_{\text {cathode }}: V_{\text {anode }}$ of BES for azo dye treatment was $3: 1$. Since the oxidation of acetate by exoelectrogenic bacteria created protons and electrons (Tice and Kim, 2014), increasing the substrate concentration could increase the effective electrons and protons for azo dye. However, the decolorization in BES-3\# with substrate concentration of $3000 \mathrm{mg} \mathrm{L}^{-1}$ was no better than that with $2000 \mathrm{mg} \mathrm{L}^{-1}$. This might be due to that the high substrate concentration might have provided continuously low local $\mathrm{pH}$ conditions near the anode surface (Tice and Kim, 2014), which inhibited the biofilm analyzes at anode, resulting in the higher electrons transfer resistance. This could be further demonstrated by the current and EIS results. In the BES-3\# with substrate concentration of 1000 , 2000 and $3000 \mathrm{mg} \mathrm{L}^{-1}$, the peak current was $0.016,0.023$ and $0.028 \mathrm{~A}$, respectively (Fig. 4B). Results showed that along with the increased substrate concentration, the peak current was increased, implying a lower polarization resistance and better electron transfer from anode to cathode, which resulted in the enhancement of electrochemical activity in this system.

The results of the impedance measurement can be presented in two common ways: Nyquist plot (Fig. 4C) and the Bode plot (Fig. 4D). Nyquist plot expresses the impedance with a real part (plotted on the $X$-axis) and an imaginary part (plotted on the $Y$-axis that is negative). The Bode plot, on the other hand, shows the information of impedance and frequency (He and Mansfeld, 2009). In this study, the Nyquist curves of the BES were typical comprised by the semicircle high frequency region and ray low frequency region. The impedance at the high frequency limit was the $R_{\mathrm{ohm}}$, the diameter of the semicircle was $R_{\mathrm{ct}}$, and the ray at low frequency region was $R_{\text {diff }}$ (You et al., 2007). EIS was closely
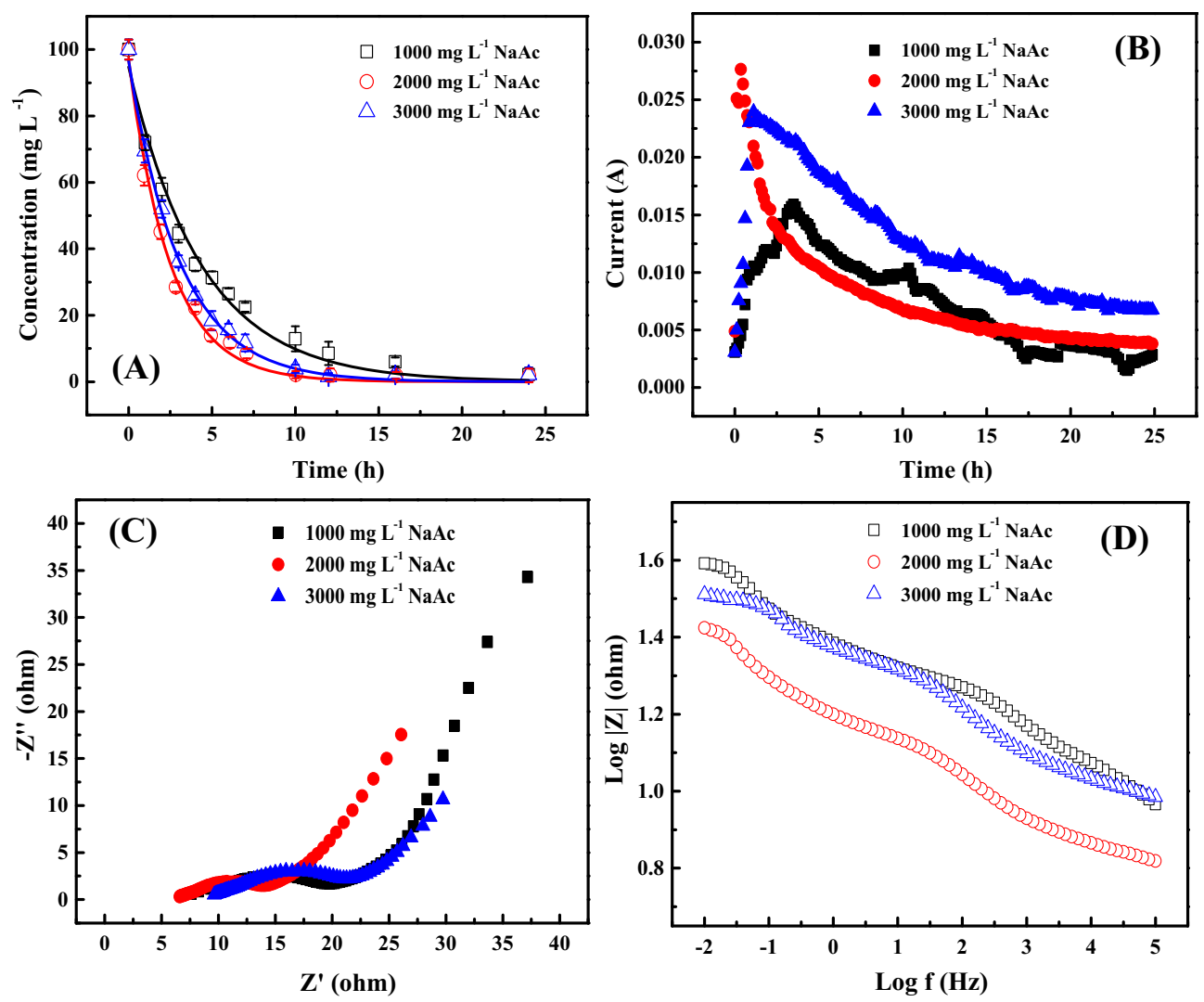

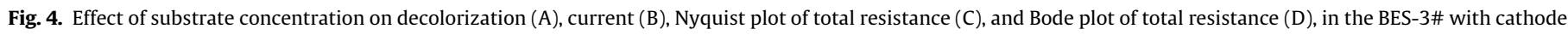
and anode volume ratio $V_{\text {cathode }}: V_{\text {anode }}=3: 1$. 
related with the reactor configuration and operation parameters. It has been reported that $R_{\text {ohm }}$ was greatly dependent on the design and configuration of the system rather than the microbial population at anode, which was predominant the total internal resistances of the system or even higher than $50 \%$ (Sekar and Ramasamy, 2013; You et al., 2008). It has demonstrated that increasing the sieve area could decrease the internal resistance and ohmic resistance to be 27.5 and $14 \Omega$, respectively (You et al., 2008). In the sleeve-type BES with three different operation conditions, the average ohmic resistances were about $7.9 \Omega$, which were relative low and only accounted for $25.4 \%$ of the average internal resistance (Fig. 4C). These results could further demonstrate that reducing the electrode spacing and increasing surface area could be effective solutions to reduce ohmic resistance (Sekar and Ramasamy, 2013). In comparison with substrate concentration of $1000 \mathrm{mg} \mathrm{L}^{-1}$, the higher concentration of $2000 \mathrm{mg} \mathrm{L}^{-1}$ showed significantly better electron transfer performance, with $R_{\mathrm{ct}}$ of $7.5 \Omega$, about $65.3 \%$ lower than that of $1000 \mathrm{mg} \mathrm{L}^{-1}(12.4 \Omega$ ) (Fig. 4C). It indicated that the biofilm at anode had enhanced electrochemical activity that could benefit to the electron transfer from microorganisms to anode. From the Bode curves (Fig. 4D), it was clearly demonstrated the better electron transfer ( $\mathrm{He}$ and Mansfeld, 2009). Moreover, the diffusion resistance for 1000 , 2000, $3000 \mathrm{mg} \mathrm{L}^{-1}$ was 20.1, 12.0 and $8.4 \Omega$, respectively (Fig. 4C). It also exhibited more and more advantages in the anolyte diffusion with increasing substrate concentration. Therefore, with the substrate concentration of $2000 \mathrm{mg} \mathrm{L}^{-1}$, BES with outer working position showed the best decolorization and reaction rate in the combined action of improving electron transfer and anolyte diffusion. It should be noted that EIS was also related with operation parameters. For example, the bacterial biofilm, mediators and electrolyte $\mathrm{pH}$ played an important role in anode impedance, meanwhile, the choices of the substrate type, substrate diffusion, mediators, cathode material type and cathode binding agent were also significant for cathode impedance (Sekar and Ramasamy, 2013).

These results demonstrated that BES with outer working position at an increased volume ratio could be further improved through increasing substrate concentration in a certain range.

\subsection{Potential and significant}

The versatility of the dual chamber BES has notably expanded the range of application for the treatment of refractory pollutants, especially contaminants those were toxic to microorganisms at anode and inhibited the effectiveness of treatment (Huang et al., 2012; Kong et al., 2015a, 2013; Mu et al., 2009a, 2009b; Tao et al., 2014). In recent years, BESs including microbial fuel cells (MFCs) and microbial electrolysis cells (MECs), have been explored extensively to treat azo dye for their innovative features and environmental benefits. In an MFC, electrical energy can be extracted from the electrical circuit. In an MEC, electrical energy needs to be supplied to the electrical circuit by a DC power supply (Mu et al., 2009a). The use of MFC takes advantage of the microbial oxidation of organic matter to generate bioelectricity simultaneously with wastewater treatment. BES can reduce the azo dye, driven by microbial oxidation of organics at the anode. It has been reported that the sleeve-type dual-chamber BES with cathode as working electrode achieved very good results in $\mathrm{Cr}$ (VI) removal and azo dye decolorization (Huang et al., 2010; Kong et al., 2013), confirming the priority of this novel configuration compared with other configurations, such as rectangular configuration and tubular configuration (Kong et al., 2014). However, it still posed a challenge on the understanding of this system and the reaction to determine the performance of BES. Taking into account that the sleeve-type configuration was composed by two tubes, including the inner chamber consisted by the inner tube, and the outer chamber consisted by the space between inner tube and outer tube, thus, the pollutants position (i.e. the working position) played an important part in the wastewater treatment. It caused the reason analysis of different effects had also become the main point in understanding and study the new configuration. Therefore, this study was performed to investigate the effect of working position in inner chamber/outer chamber on decolorization of azo dye.

Results indicated that the cathode in outer chamber performed better decolorization compared to that in inner chamber at the cathode and anode chamber volume ratio of $1: 1$. To further investigate the effect of amplified cathode volume ratio, BES reactors with $V_{\text {cathode }}: V_{\text {anode }}$ of $3: 1$ were also compared. It showed that the working position in outer chamber was also much better than inner chamber. EIS results demonstrated that when the cathode was placed in the outer chamber (BES-1\# and BES-3\#), the total resistance of BES was decreased, as a result of decreased anode resistance and cathode resistance (Figs. $2 \mathrm{C}$ and $3 \mathrm{C}$ ). Moreover, the detailed analysis of components in anode resistance showed the improvement of charge transfer resistance and diffusion resistance in BES with outer working position (Figs. 2D and 3D). So it can be concluded that sleeve-type BES with outer cathode and inner anode was more conducive to facilitate the degradation of organic matter at anode, and made effective delivery of electrons and protons to the cathode, finally resulting in the better decolorization (Figs. 2A and 3A). This can also be demonstrated by the reduced cathode resistance in BES (Figs. $2 \mathrm{D}$ and $3 \mathrm{D}$ ). It should be noted that with the increased cathode volume ratio, the performance was inhibited, possibly for the inadequate electrons supply with the decreasing anode volume at the same substrate concentration. It could be further improved by increasing the substrate concentration in anode chamber, through reducing the charge transfer resistance and diffusion resistance (Fig. 4C), leading to increased decolorization rate (Fig. 4A).

This study not only investigated the optimization of working position in outer chamber or inner chamber, but also evaluated and optimized the working electrode position in increasing volume of cathode chamber. It has very important significance in configuration optimization and will promote the application of sleevetype BES in wastewater treatment. It is expected that the broad platform described here will stimulate new thoughts and ideas which can then develop into a new generation of BES application.

\section{Conclusion}

This study demonstrated that the outer working position in the sleeve-type BES took obvious priority in azo dye decolorization with the same volume ratio of cathode and anode $(1: 1$ or $3: 1)$. The performance with increased volume ratio could be further improved through increasing the substrate supply. It provided the basic theory for the optimization of BES with sleeve-type configuration and would have a great potential in the application.

\section{Acknowledgements}

This research was supported by the National Science Foundation for Distinguished Young Scholars (No. 51225802), the Shanghai Tongji Gao Tingyao Environmental Science \& Technology Development Foundation (STGEF), and the National High-tech R\&D Program of China (No. 2012AA051502).

\section{References}

Ge, Z., Dosoretz, C.G., He, Z., 2014. Effects of number of cell pairs on the performance of microbial desalination cells. Desalination 341, 101-106. 
Gil-Carrera, L., Mehta, P., Escapa, A., Moran, A., Garcia, V., Guiot, S.R., Tartakovsky, B. 2011. Optimizing the electrode size and arrangement in a microbial electrolysis cell. Bioresour. Technol. 102, 9593-9598.

He, Z., Mansfeld, F., 2009. Exploring the use of electrochemical impedance spectroscopy (EIS) in microbial fuel cell studies. Energy Environ. Sci. 2, 215-219.

Huang, L.P., Chen, J.W., Quan, X., Yang, F.L., 2010. Enhancement of hexavalent chromium reduction and electricity production from a biocathode microbial fuel cell. Bioprocess Biosyst. Eng. 33, 937-945.

Huang, L.P., Chai, X.L., Quan, X., Logan, B.E., Chen, G.H., 2012. Reductive dechlorination and mineralization of pentachlorophenol in biocathode microbial fuel cells. Bioresour. Technol. 111, 167-174.

Jacobson, K.S., Drew, D.M., He, Z., 2011. Use of a liter-scale microbial desalination cell as a platform to study bioelectrochemical desalination with salt solution or artificial seawater. Environ. Sci. Technol. 45, 4652-4657.

Kashyap, D., Dwivedi, P.K., Pandey, J.K., Kim, Y.H., Kim, G.M., Sharma, A., Goel, S., 2014. Application of electrochemical impedance spectroscopy in bio-fuel cell characterization: a review. Int. J. Hydrogen Energy 39, 20159-20170.

Kong, F., Wang, A., Liang, B., Liu, W., Cheng, H., 2013. Improved azo dye decolorization in a modified sleeve-type bioelectrochemical system. Bioresour. Technol. 143, 669-673.

Kong, F., Wang, A., Cheng, H., Liang, B., 2014. Accelerated decolorization of azo dye Congo red in a combined bioanode-biocathode bioelectrochemical system with modified electrodes deployment. Bioresour. Technol. 151, 332-339.

Kong, D., Liang, B., Yun, H., Cheng, H., Ma, J., Cui, M., Wang, A., Ren, N., 2015a. Cathodic degradation of antibiotics: characterization and pathway analysis. Water Res. 72, 281-292.

Kong, F., Wang, A., Ren, H.-Y., 2015b. Improved azo dye decolorization in an advanced integrated system of bioelectrochemical module with surrounding electrode deployment and anaerobic sludge reactor. Bioresour. Technol. 175, 624-628.

Kong, F., Wang, A., Ren, H.-Y., 2015c. Optimized matching modes of bioelectrochemical module and anaerobic sludge in the integrated system for azo dye treatment. Bioresour. Technol. 192, 486-493.

Li, W.-W., Yu, H.-Q., He, Z., 2014. Towards sustainable wastewater treatment by using microbial fuel cells-centered technologies. Energy Environ. Sci. 7, 911924

Liang, B., Cheng, H.-Y., Kong, D.-Y., Gao, S.-H., Sun, F., Cui, D., Kong, F.-Y., Zhou, A.-J., Liu, W.-Z., Ren, N.-Q., 2013. Accelerated reduction of chlorinated nitroaromatic antibiotic chloramphenicol by biocathode. Environ. Sci. Technol. 47, 53535361.

Montpart, N., Rago, L., Baeza, J.A., Guisasola, A., 2015. Hydrogen production in single chamber microbial electrolysis cells with different complex substrates. Water Res. 68, 601-615.

Mu, Y., Rabaey, K., Rozendal, R.A., Yuan, Z., Keller, J., 2009a. Decolorization of azo dyes in bioelectrochemical systems. Environ. Sci. Technol. 43, 5137-5143.

Mu, Y., Rozendal, R.A., Rabaey, K., Keller, J., 2009b. Nitrobenzene removal in bioelectrochemical systems. Environ. Sci. Technol. 43, 8690-8695.
Ping, Q., He, Z., 2013. Improving the flexibility of microbial desalination cells through spatially decoupling anode and cathode. Bioresour. Technol. 144, $304-$ 310.

Raman, K., Lan, J.C.W., 2012. Performance and kinetic study of photo microbial fue cells (PMFCs) with different electrode distances. Appl. Energy 100, 100-105.

Sekar, N., Ramasamy, R.P., 2013. Electrochemical impedance spectroscopy for microbial fuel cell characterization. J. Microb. Biochem. Technol. S 6, 2.

Sleutels, T.H.J.A., Ter Heijne, A., Buisman, C.J.N., Hamelers, H.V.M., 2012 Bioelectrochemical systems: an outlook for practical applications. ChemSusChem 5, 1012-1019.

Tao, H.-C., Lei, T., Shi, G., Sun, X.-N., Wei, X.-Y., Zhang, L.-J., Wu, W.-M., 2014 Removal of heavy metals from fly ash leachate using combined bioelectrochemical systems and electrolysis. J. Hazard. Mater. 264, 1-7.

Tice, R.C., Kim, Y., 2014. Influence of substrate concentration and feed frequency on ammonia inhibition in microbial fuel cells. J. Power Sources 271, 360-365.

Uría, N., Sánchez, D., Mas, R., Sánchez, O., Muñoz, F.X., Mas, J., 2012. Effect of the cathode/anode ratio and the choice of cathode catalyst on the performance of microbial fuel cell transducers for the determination of microbial activity Sensors Actuators B: Chem. 170, 88-94.

Velasquez-Orta, S.B., Head, I.M., Curtis, T.P., Scott, K., 2011. Factors affecting current production in microbial fuel cells using different industrial wastewaters. Bioresour. Technol. 102, 5105-5112.

Wang, H., Ren, Z.J., 2013. A comprehensive review of microbial electrochemical systems as a platform technology. Biotechnol. Adv. 31, 1796-1807.

Wang, A.-J., Cheng, H.-Y., Liang, B., Ren, N.-Q., Cui, D., Lin, N., Kim, B.H., Rabaey, K. 2011. Efficient reduction of nitrobenzene to aniline with a biocatalyzed cathode. Environ. Sci. Technol. 45, 10186-10193.

Wang, Z., Mei, X., Ma, J., Wu, Z., 2012. Recent advances in microbial fuel cells integrated with sludge treatment. Chem. Eng. Technol. 35, 1733-1743.

Wang, Y.-H., Wang, B.-S., Liu, Y.-P., Chen, Q.-Y., 2013. Electricity and hydrogen coproduction from a bio-electrochemical cell with acetate substrate. Int. J. Hydrogen Energy 38, 6600-6606.

You, S.J., Zhao, O.L., Zhang, J.N., Jiang, J.Q., Wan, C.L., Du, M.A., Zhao, S.O., 2007. A graphite-granule membrane-less tubular air-cathode microbial fuel cell for power generation under continuously operational conditions. J. Power Sources $173,172-177$.

You, S.J., Zhao, Q.L., Zhang, J., Liu, H., Jiang, J.Q., Zhao, S.Q., 2008. Increased sustainable electricity generation in up-flow air-cathode microbial fuel cells. Biosens. Bioelectron. 23, 1157-1160.

Zhao, F., Slade, R.C.T., Varcoe, J.R., 2009. Techniques for the study and development of microbial fuel cells: an electrochemical perspective. Chem. Soc. Rev. 38, 1926-1939.

Zille, A., Ramalho, P., Tzanov, T., Millward, R., Aires, V., Cardoso, M.H., Ramalho, M.T. Gübitz, G.M., Cavaco-Paulo, A., 2004. Predicting dye biodegradation from redox potentials. Biotechnol. Prog. 20, 1588-1592. 